\title{
A Síndrome de Burnout e suas repercussões no cotidiano de trabalho do enfermeiro
}

\author{
Burnout Syndrome and its repercussions on nurses' daily work \\ El síndrome de Burnout y sus repercusiones en el trabajo diario del enfermero
}

Recebido: 28/03/2021 | Revisado: 02/04/2021 | Aceito: 04/04/2021 | Publicado: 15/04/2021

\author{
Ruy de Almeida Barcellos \\ ORCID: https://orcid.org/0000-0002-7857-2151 \\ Hospital de Clínicas de Porto Alegre, Brasil \\ E-mail: rbarcellos@hcpa.edu.br \\ Maria Angélica Gazzana de Lucena \\ ORCID: https://orcid.org/0000-0001-8558-0785 \\ Enfermeira. Mestra em Filosofia, Brasil \\ E-mail: angelica.gazzana@gmail.com \\ Juliana Langendorf da Costa Vieira \\ ORCID: https://orcid.org/0000-0003-0089-4633 \\ Universidade Federal do Rio Grande do Sul, Brasil \\ E-mail: julanghi@gmail.com
}

\begin{abstract}
Resumo
Objetivo: Identificar as principais manifestações da Síndrome de Burnout em Enfermeiros Intensivistas. Metodologia: Trata-se de um estudo descritivo realizado através da aplicação do Inventário de Burnout de Maslach (MBI). Realizou-se em três Unidades de Terapia Intensiva adulto e uma neonatal de uma instituição hospitalar filantrópica de grande porte. Participaram da pesquisa dezoito enfermeiros que desenvolvem atividades nas unidades participantes. Resultados: A população pesquisada se mostrou motivada e satisfeita com o trabalho, reconhecendo a importância do trabalho que realizam. Conclusão: Os resultados obtidos permitiram constatar que, apesar do trabalho ser considerado extenuante e existir indícios de esgotamento por parte dos profissionais, não houve uma grande carga de exaustão profissional.
\end{abstract}

Palavras-chave: Esgotamento profissional; Enfermeiros; Unidades de Terapia Intensiva.

\begin{abstract}
Objective: To identify the main manifestations of Burnout Syndrome in Intensive Care Nurses. Methodology: This is a descriptive study carried out through the application of the Maslach Burnout Inventory (MBI). It was carried out in three adult intensive care units and one neonatal unit in a large philanthropic hospital. Eighteen nurses participated in the research, who carry out activities in the participating units. Results: The researched population was motivated and satisfied with the work, recognizing the importance of the work they do. Conclusion: The results obtained showed that, despite the work being considered strenuous and there are signs of exhaustion on the part of the professionals, there was not a great deal of professional exhaustion.
\end{abstract}

Keywords: Burnout; Nurses; Intensive Care Units.

\section{Resumen}

Objetivo: Identificar las principales manifestaciones del Síndrome de Burnout en Enfermeros de Cuidados Intensivos. Metodología: Se trata de un estudio descriptivo realizado mediante la aplicación del Maslach Burnout Inventory (MBI). Se llevó a cabo en tres unidades de cuidados intensivos para adultos y una unidad neonatal en un gran hospital filantrópico. En la investigación participaron dieciocho enfermeros, quienes realizan actividades en las unidades participantes. Resultados: La población investigada se mostró motivada y satisfecha con el trabajo, reconociendo la importancia del trabajo que realizan. Conclusión: Los resultados obtenidos mostraron que, a pesar de que el trabajo se considera arduo y hay signos de agotamiento por parte de los profesionales, no existe un gran agotamiento profesional.

Palabras clave: Agotamiento profesional; Enfermeras y Enfermeros; Unidades de Cuidados Intensivos.

\section{Introdução}

A Síndrome de Burnout é considerada um fenômeno social, uma expressão de tempos de crise e desorientação da sociedade atual observada a partir de constantes incertezas e insatisfações com o modo de vida e trabalho. A síndrome, também conhecida como Síndrome de esgotamento profissional, afeta principalmente aqueles profissionais que prestam assistência, 
visto que cada vez mais se exige, por parte dos usuários, que este profissional tenha capacidade de resolver todos os problemas e que seja movido pelo sentimento de compaixão (Perniciotti et al, 2020; Carlotto \& Câmara, 2008).

Geralmente, o burnout é uma reação à tensão crônica por se lidar direta e constantemente com pessoas. Essa síndrome afeta principalmente profissionais da área de serviços ou cuidadores que estão em contato direto e permanente com os usuários, como os trabalhadores da saúde e da educação (França \& Ferrari, 2012)

A síndrome de Burnout é uma condição em que o trabalhador perde o sentido da sua relação com o trabalho, de forma que as coisas já não importam mais e qualquer esforço lhe parece inútil. Tem um conceito multidimensional que envolve três componentes, a saber: a exaustão emocional; a despersonalização e a falta de envolvimento pessoal no trabalho. Encontra-se na literatura especializada algumas diferenças entre os autores no que diz respeito à sequência de encadeamento dos eventos negativos e do significado das suas manifestações. No entanto, considera-se que os componentes da síndrome, em suas dimensões propostas, são fundamentais no burnout. (Silva et al, 2020; Tamayo \& Tróccoli, 2002).

De sua sintomatologia clínica podem ser observadas manifestações físicas como fadiga constante, distúrbios do sono, falta de apetite, dores musculares generalizadas ou difusas. As manifestações psíquicas caracterizam-se por falta de atenção, alterações na memória, ansiedade e sentimento de frustração. As alterações comportamentais são identificadas através da negligência no trabalho, irritabilidade, diminuição da capacidade de concentração, conflitos com colegas, necessidade de longas pausas para o descanso e o cumprimento irregular da carga horária de trabalho. O comportamento defensivo ocorre quando o trabalhador apresenta tendência ao isolamento, sentimento de onipotência e empobrecimento da qualidade do trabalho prestado (Braga \& Paula, 2018; Carlotto \& Câmara, 2008).

Um dos instrumentos mais utilizados em todo o mundo para avaliar essa síndrome é o Maslach Burnout Inventory (MBI). Elaborado em 1978, o MBI constitui um questionário autoaplicável que avalia índices de burnout de acordo com os escores de cada dimensão, sendo que altos escores em exaustão emocional e despersonalização e baixos escores em realização profissional indicam alto nível de burnout (Maslach \& Jackson, 1981). De acordo com Dutra (2019), a versão atual apresenta 22 itens. Desses, 9 são relativos à dimensão exaustão emocional (EE), que pretende analisar sentimentos de sobrecarga emocional; 8 à despersonalização (DE), que pretende medir respostas "frias", impessoais, negativas dirigidas àqueles a quem se presta atendimento; e 5 referem-se à realização profissional (RP), usada para avaliar sentimentos de incompetência e baixa ou falta de realização (Dutra et al, 2019).

Do exposto, ao buscar a compreensão de uma realidade, acredita-se de suma importância o reconhecimento dessa situação no cotidiano de trabalho dos Enfermeiros locados em Unidade de Terapia Intensiva (UTI). Nesse sentido, o objetivo dessa pesquisa foi o de identificar a presença de manifestações da Síndrome de Burnout em Enfermeiros locados em Unidades de Terapia Intensiva de um hospital de grande porte.

\section{Metodologia}

Trata-se de um estudo descritivo quali-quantitativo conforme proposto por Estrela (2018), realizado em uma instituição hospitalar filantrópica de grande porte, localizada na cidade de Caxias do Sul (RS). O estudo ocorreu em três UTI adulto e uma neonatal. A população pesquisada foi constituída estritamente por Enfermeiros que exerciam a assistência nestas unidades.

A amostragem ocorreu por conveniência e constituiu-se por um total de dezoito (18) Enfermeiros, sendo dois (2) pertencentes à Unidade de Terapia Intensiva Neonatal e os demais (16) da UTI adulto. A presente pesquisa obedeceu ao cumprimento dos princípios éticos e foi aprovada pelo Comitê de Ética em Pesquisa da Instituição Hospitalar sob parecer número 1.589.212. Os pesquisados foram informados sobre a pesquisa, cada um deles recebendo o Termo de Consentimento Livre Esclarecido contendo informações sobre as implicações do estudo. Após consentimento, os participantes receberam o 
questionário autoaplicável e anônimo, com um agendamento de sua devolução conforme disponibilidade local. A coleta de dados ocorreu através da aplicação de um questionário para o levantamento dos dados sociodemográficos e do Inventário de Burnout de Maslach (MBI).

A análise dos dados se deu através da estatística descritiva com a apresentação de números absolutos e percentuais discriminados em forma de tabelas com escala do tipo Likert, com escala ordinal variando de 1 a 7 (1-nunca, 2-algumas vezes por ano, 3-uma vez por mês, 4-algumas vezes por mês, 5-uma vez por semana, 6-algumas vezes por semanas e 7-todos os dias). O somatório das dimensões relativas ao MBI foi comparado com os valores de referência.

\section{Resultados e Discussão}

Para uma primeira parte, procedeu-se à análise descritiva geral referente à caracterização sociodemográfica da população do estudo. Conforme apresentado na Tabela 1, os participantes pesquisados totalizaram 18 Enfermeiros, com tempo de experiência igual ou maior há dois anos. A prevalência foi do sexo feminino (100\%), com faixa etária preponderante entre 31 a 35 anos ( $38,8 \%)$, sendo a maioria (66\%) das pesquisadas casadas.

No que tange a maior presença de mulheres no quadro pesquisado, acredita-se estar essa característica relacionada à significativa inserção da mulher no mercado de trabalho, de maneira geral, como também a uma denominada feminização da Enfermagem, já histórica. Fato esse apresentado por muitas pesquisas na área, como também na mais recente pesquisa realizada pela FIOCRUZ - COFEN sobre o Perfil da Enfermagem no Brasil (com Enfermeiros, Técnicos e Auxiliares de Enfermagem), com resultados que apontaram à presença do sexo feminino $(85,1 \%)$ de forma mais destacada (sexo masculino foi de 14,4\%), (Machado, 2017; Barbosa et al, 2019).

Em relação à carga de trabalho essa se distribuiu entre 6 a 12 horas diárias, perfazendo 36 horas semanais, em turno manhã, tarde ou noite, que compreendeu a quase totalidade dos pesquisados $16(88,8 \%)$. Há os que exercem o turno integral (manhã e tarde), em regime de 40 horas semanais $2(11,1 \%)$. Apenas um $(5,5 \%)$ deles exercia suas atividades em outro local de trabalho, perfazendo um total de 16 horas semanais. Outra variável analisada, em relação ao estado civil, como a encontrada nesta pesquisa e ao encontro da literatura especializada, se atribui ao casamento ou à situação de companheiro(a) estável e ao fato de ter filhos uma menor propensão ao burnout. (Haddad \& Jodas, 2009). 
Tabela 1. Características sociodemográficas da amostra.

\begin{tabular}{|c|c|c|}
\hline Variáveis & n (18) & $\%$ \\
\hline \multicolumn{3}{|l|}{ Sexo } \\
\hline Feminino & 18 & 100 \\
\hline \multicolumn{3}{|l|}{ Faixa etária (anos) } \\
\hline$<30$ & 6 & 33,3 \\
\hline 34 a 35 & 7 & 38,8 \\
\hline 36 a 40 & 3 & 16,6 \\
\hline$>45$ & 2 & 11,1 \\
\hline \multicolumn{3}{|l|}{ Estado Civil } \\
\hline Solteiro & 5 & 27,7 \\
\hline Casado & 12 & 66,6 \\
\hline Divorciado & 1 & 5,5 \\
\hline \multicolumn{3}{|c|}{ Carga de trabalho $\backslash$ Horas semanais } \\
\hline $36 \mathrm{~h}$ & 16 & 88,8 \\
\hline $40 \mathrm{~h}$ & 2 & 11,1 \\
\hline
\end{tabular}

Fonte: Dados da pesquisa.

Em relação propriamente às dimensões da Síndrome de Burnout, a exaustão emocional (EE) é tida como a primeira resposta ao estresse laboral crônico, acompanhado de desgaste físico e esgotamento dos recursos emocionais para lidar com a situação estressora. Apresenta-se a sua ocorrência entre os Enfermeiros das UTI pesquisadas na Tabela 2.

Observou-se que dos 18 Enfermeiros entrevistados, 6 (33,33\%) se dizem estimulados pela clientela, como também, em igual número, lidam bem com os problemas no cotidiano do trabalho. Desses, 2 (11,11\%) encontram-se no limite e emocionalmente exaustos e apenas $1(5,56 \%)$ esgotado ao final do dia.

Nenhum deles mostrou-se frustrado com o trabalho, nem estressado por trabalhar com pessoas. Assim, pelas médias obtidas, os resultados revelam que nesse grupo de profissionais Enfermeiros não há uma grande carga de exaustão profissional.

Estudos identificam a satisfação no trabalho como um fator de proteção ao burnout. Por outro lado, a sobrecarga de trabalho representa uma fonte de estresse crônico entre Enfermeiros, constituindo-se num dos principais preditores da exaustão emocional (Rodrigues, Santos, \& Sousa, 2017; Silva et al., 2019; Rosa \& Carlotto, 2005; Benevides - Pereira, 2014). 
Tabela 2. Dimensão Exaustão Profissional.

\begin{tabular}{|c|c|c|c|c|c|c|c|c|c|c|c|c|c|c|}
\hline & \multicolumn{2}{|c|}{ Nunca } & \multicolumn{2}{|c|}{$\begin{array}{l}\text { Algumas } \\
\text { vezes/ano }\end{array}$} & \multicolumn{2}{|c|}{$\begin{array}{c}\text { Uma } \\
\text { vez/mês }\end{array}$} & \multicolumn{2}{|c|}{$\begin{array}{c}\text { Algumas } \\
\text { vezes/mês }\end{array}$} & \multicolumn{2}{|c|}{$\begin{array}{c}\text { Uma } \\
\text { vez/semana }\end{array}$} & \multicolumn{2}{|c|}{$\begin{array}{c}\text { Algumas } \\
\text { vezes/sem } \\
\text { ana }\end{array}$} & \multicolumn{2}{|c|}{$\begin{array}{l}\text { Todos os } \\
\text { dias }\end{array}$} \\
\hline & $\mathbf{n}$ & $\%$ & $\mathbf{n}$ & $\%$ & $\mathbf{n}$ & $\%$ & $\mathbf{n}$ & $\%$ & $\mathbf{n}$ & $\%$ & $\mathbf{n}$ & $\%$ & $\mathbf{n}$ & $\%$ \\
\hline $\begin{array}{l}\text { Cheio de } \\
\text { energia }\end{array}$ & 0 & - & 0 & - & 2 & 11,11 & 3 & 16,67 & 1 & 5,56 & 12 & 66,67 & 0 & - \\
\hline $\begin{array}{l}\text { Esgotado final } \\
\text { do dia }\end{array}$ & 1 & 5,56 & 1 & 5,56 & 1 & 5,56 & 1 & 5,56 & 3 & 16,67 & 11 & 61,11 & 1 & 5,56 \\
\hline $\begin{array}{l}\text { Culpado pela } \\
\text { clientela }\end{array}$ & 4 & 22,22 & 7 & 38,89 & 1 & 5,56 & 2 & 11,11 & 1 & 5,56 & 3 & 16,67 & 0 & - \\
\hline $\begin{array}{c}\text { Estimulado } \\
\text { pela clientela }\end{array}$ & 0 & - & 1 & 5,56 & 0 & - & 6 & 33,33 & 1 & 5,56 & 4 & 22,22 & 6 & $\begin{array}{c}\mathbf{3 3}, 3 \\
\mathbf{3}\end{array}$ \\
\hline Final do limite & 2 & 11,11 & 5 & 27,78 & 1 & 5,56 & 5 & 27,78 & 1 & 5,56 & 2 & 11,11 & 2 & $\begin{array}{c}11,1 \\
1\end{array}$ \\
\hline $\begin{array}{l}\text { Lida com os } \\
\text { problemas }\end{array}$ & 0 & - & 0 & - & 0 & - & 2 & 11,11 & 4 & 22,22 & 6 & 33,33 & 6 & $\begin{array}{c}\mathbf{3 3}, \mathbf{3} \\
\mathbf{3}\end{array}$ \\
\hline $\begin{array}{l}\text { Emocionalmen } \\
\text { te exausto }\end{array}$ & 2 & 11,11 & 5 & 27,78 & 1 & 5,56 & 4 & 22,22 & 1 & 5,56 & 3 & 16,67 & 2 & $\begin{array}{c}11,1 \\
1\end{array}$ \\
\hline $\begin{array}{l}\text { Frustrado com } \\
\text { o trabalho }\end{array}$ & 2 & 11,11 & 9 & 50,00 & 1 & 5,56 & 3 & 16,67 & 2 & 11,11 & 1 & 5,56 & 0 & - \\
\hline $\begin{array}{l}\text { Estressado por } \\
\text { trabalhar com } \\
\text { pessoas }\end{array}$ & 3 & 16,67 & 7 & 38,89 & 2 & 11,11 & 2 & 11,11 & 3 & 16,67 & 1 & 5,56 & 0 & - \\
\hline
\end{tabular}

Fonte: dados da pesquisa.

Seguindo as dimensões trabalhadas e seus indicadores, segue à dimensão que avalia a Despersonalização (D), entendida por alguns por "desumanização", a qual é marcada pelo distanciamento emocional e indiferença em relação ao trabalho ou aos usuários do serviço.

A Tabela 3 mostra os resultados obtidos. Nela pode-se observar que dos Enfermeiros pesquisados, 6 (33,33\%) entendem que conseguem criar um ambiente tranquilo com a clientela, assim como, em igual número, $6(33,33 \%)$ influenciam positivamente outras pessoas. Por outro lado, 3 (16,67\%) dos Enfermeiros sentem que estão trabalhando demais e 2 (11,11\%) esgotados em função do trabalho, de modo contínuo. Diante desse indício de esgotamento se considera importante acompanhar a equipe através de uma monitorização e políticas de ações de saúde do trabalhador.

A despersonalização pode funcionar como estratégia defensiva por distanciar o indivíduo do convívio mais próximo com usuários, acarretar conflitos no relacionamento interpessoal e prejudicar a própria imagem profissional (Benevides Pereira, 2014; Dalmolin et al., 2014). 
Tabela 3. Dimensão Despersonalização.

\begin{tabular}{|c|c|c|c|c|c|c|c|c|c|c|c|c|c|c|}
\hline & \multicolumn{2}{|c|}{ Nunca } & \multicolumn{2}{|c|}{$\begin{array}{l}\text { Algumas } \\
\text { vezes/ano }\end{array}$} & \multicolumn{2}{|c|}{$\begin{array}{c}\text { Uma } \\
\text { vez/mês }\end{array}$} & \multicolumn{2}{|c|}{$\begin{array}{l}\text { Algumas } \\
\text { vezes } / \mathbf{m e ̂} \\
\mathbf{s}\end{array}$} & \multicolumn{2}{|c|}{$\begin{array}{c}\text { Uma } \\
\text { vez/seman } \\
\text { a }\end{array}$} & \multicolumn{2}{|c|}{$\begin{array}{c}\text { Algumas } \\
\text { vezes/sema } \\
\text { na }\end{array}$} & \multicolumn{2}{|c|}{$\begin{array}{c}\text { Todos os } \\
\text { dias }\end{array}$} \\
\hline & $\mathbf{n}$ & $\%$ & $\mathbf{n}$ & $\%$ & $\mathbf{n}$ & $\%$ & $\mathbf{n}$ & $\%$ & $\mathbf{n}$ & $\%$ & $\mathbf{n}$ & $\%$ & n & $\%$ \\
\hline $\begin{array}{l}\text { Esgotado com } \\
\text { trabalho }\end{array}$ & 3 & 16,67 & 6 & 33,33 & 2 & 11,11 & 2 & 11,11 & 1 & 5,56 & 2 & 11,11 & 2 & 11,11 \\
\hline $\begin{array}{l}\text { Cria ambiente } \\
\text { tranqüilo com } \\
\text { a clientela }\end{array}$ & 1 & 5,56 & 1 & 5,56 & 1 & 5,56 & 2 & 11,11 & 3 & 16,67 & 4 & 22,22 & 6 & 33,33 \\
\hline $\begin{array}{l}\text { Influencia } \\
\text { positivamente } \\
\text { outras pessoas }\end{array}$ & 1 & 5,56 & 3 & 16,67 & 1 & 5,56 & 3 & 16,67 & 2 & 11,11 & 2 & 11,11 & 6 & $\mathbf{3 3}, \mathbf{3 3}$ \\
\hline $\begin{array}{l}\text { Tratam } \\
\text { clientes como } \\
\text { objetos }\end{array}$ & $\begin{array}{l}1 \\
4\end{array}$ & 77,78 & 1 & 5,56 & 0 & - & 1 & 5,56 & 0 & - & 2 & 11,11 & 0 & - \\
\hline $\begin{array}{l}\text { Sente que está } \\
\text { trabalhando } \\
\text { demais }\end{array}$ & 3 & 16,67 & 3 & 16,67 & 2 & 11,11 & 6 & 33,33 & 0 & - & 1 & 5,56 & 3 & 16,67 \\
\hline
\end{tabular}

Fonte: Dados da pesquisa.

Já no que diz respeito à dimensão que avalia a Realização Profissional (RP), considera-se que a diminuição da realização pessoal é evidenciada pela ausência de perspectivas futuras, frustração e sentimentos de incompetência e fracasso. A baixa realização no trabalho é representada pela tendência do trabalhador em se autoavaliar de forma negativa. Ele torna-se insatisfeito com o seu desenvolvimento profissional, além de apresentar uma diminuição no sentimento de competência e êxito. É comum o sentimento de "não valer a pena” (Sá, Martins-Silva \& Funchal, 2014; Carlotto \& Câmara, 2008).

Os resultados obtidos referentes a essa dimensão avaliada, juntamente com a descrição dos itens, são apresentados na Tabela 4, de onde se extrai que 14 (77,78\%) dos pesquisados consideraram que realizam feitos importantes no trabalho, seguidos por $10(55,56 \%)$ que julgaram tratar adequadamente os problemas da clientela a que se encontram responsáveis.

Dos participantes, 6 (33,33\%) afirmaram que entendem facilmente o que sua clientela sente. Em contrapartida, 4 $(22,22 \%)$ sentem-se cansados, como também, em igual número, 4 (22,22\%) percebem-se endurecendo emocionalmente. Do total de pesquisados, $3(16,67 \%)$ registraram que ficaram insensíveis após terem começado a trabalhar em UTI e $1(5,56 \%)$ registra que trabalhar com pessoas exige esforço. Nenhum deles, no entanto, considerou como alternativa que não se importa com a clientela.

Jornadas de trabalho prolongadas somadas à excessiva carga de trabalho acarretam ao profissional sintomas de fadiga, esgotamento físico e mental, além de sentimentos de agressividade e desprezo com a equipe, provocando consequências negativas ao cuidado destinado ao paciente, bem como, a vida pessoal, social e ocupacional (Rodrigues, Santos \& Sousa, 2017; Silva et al, 2019).

O sentimento de RP pode funcionar como um fator protetor no desencadeamento do burnout e estar relacionado às recompensas como: a possibilidade de crescimento na carreira, o respeito da chefia e colegas de trabalho, o apoio das pessoas em momentos difíceis e tratamento justo. Desta forma, quando muito esforço é recompensado surgem sentimentos como satisfação, motivação e diminuem os riscos de estresse e adoecimento. Portanto, o trabalho por ser fundamental e 
complementar a vida humana deve estar relacionado ao alcance de objetivos pessoais, à necessidade de envolvimento do indivíduo e à motivação para que seja atribuído sentido à atividade (Barboza et al, 2018).

Tabela 4. Dimensão Realização Profissional.

\begin{tabular}{|c|c|c|c|c|c|c|c|c|c|c|c|c|c|c|}
\hline & \multicolumn{2}{|c|}{ Nunca } & \multicolumn{2}{|c|}{$\begin{array}{c}\text { Algumas } \\
\text { vezes/ano }\end{array}$} & \multicolumn{2}{|c|}{$\begin{array}{c}\text { Uma } \\
\text { vez/mês }\end{array}$} & \multicolumn{2}{|c|}{$\begin{array}{l}\text { Algumas } \\
\text { vezes/mês }\end{array}$} & \multicolumn{2}{|c|}{$\begin{array}{c}\text { Uma } \\
\text { vez/seman } \\
\text { a }\end{array}$} & \multicolumn{2}{|c|}{$\begin{array}{c}\text { Algumas } \\
\text { vezes/sema } \\
\text { na }\end{array}$} & \multicolumn{2}{|c|}{$\begin{array}{l}\text { Todos os } \\
\text { dias }\end{array}$} \\
\hline & $\mathbf{n}$ & $\%$ & $\mathbf{n}$ & $\%$ & $\mathbf{n}$ & $\%$ & $\mathbf{n}$ & $\%$ & $\mathbf{n}$ & $\%$ & $\mathbf{n}$ & $\%$ & $\mathbf{n}$ & $\%$ \\
\hline $\begin{array}{l}\text { Trata adequadamente } \\
\text { problemas da } \\
\text { clientela }\end{array}$ & 0 & - & 0 & - & 1 & 5,56 & 1 & 5,56 & 2 & 11,11 & 4 & 22,22 & 10 & 55,56 \\
\hline Sinto-me cansado & 1 & 5,56 & 6 & 33,33 & 1 & 5,56 & 3 & 16,67 & 1 & 5,56 & 2 & 11,11 & 4 & 22,22 \\
\hline $\begin{array}{l}\text { Trabalhar com } \\
\text { pessoas é um esforço }\end{array}$ & 5 & 27,78 & 8 & 44,44 & 1 & 5,56 & 2 & 11,11 & 0 & - & 1 & 5,56 & 1 & 5,56 \\
\hline $\begin{array}{l}\text { Entendo facilmente o } \\
\text { que sente minha } \\
\text { clientela }\end{array}$ & 0 & - & 1 & 5,56 & 0 & - & 2 & 11,11 & 1 & 5,56 & 7 & 38,89 & 6 & 33,33 \\
\hline $\begin{array}{l}\text { Fiquei insensível } \\
\text { após começar } \\
\text { trabalhar }\end{array}$ & 9 & 50,00 & 3 & 16,67 & 0 & - & 3 & 16,67 & 0 & - & 0 & - & 3 & 16,67 \\
\hline $\begin{array}{l}\text { Endurecendo } \\
\text { emocionalmente }\end{array}$ & 5 & 27,78 & 4 & 22,22 & 2 & 11,11 & 3 & 16,67 & 0 & - & 0 & - & 4 & 22,22 \\
\hline $\begin{array}{l}\text { Não me importo com } \\
\text { a clientela }\end{array}$ & 15 & 83,33 & 2 & 11,11 & 0 & - & 0 & - & 0 & - & 1 & 5,56 & 0 & - \\
\hline $\begin{array}{l}\text { Realizo coisas } \\
\text { importantes no meu } \\
\text { trabalho }\end{array}$ & 0 & - & 2 & 11,11 & 0 & - & 0 & - & 1 & 5,56 & 1 & 5,56 & 14 & 77,78 \\
\hline
\end{tabular}

Fonte: Dados da pesquisa.

A leitura dos resultados apresentados por esse estudo revelou não haver associação entre as dimensões do burnout com os pesquisados, uma vez que se considera com burnout uma pessoa que apresenta altas pontuações em $E E$ e $D E$ associadas a baixos valores em $R P$.

A população pesquisada se mostrou motivada e satisfeita com o trabalho e reconheceu a importância do trabalho que realizam, bem como se importam com a clientela. A percepção da utilidade do próprio trabalho tem valor inegável para a autoestima do trabalhador.

Quanto ao ambiente de trabalho, acredita-se que unidades de alta complexidade e sobrecarga de trabalho influenciam no acometimento psicológico desses profissionais. A UTI, unidade de referência dos pesquisados, é considerada um dos ambientes hospitalares mais exaustivos, agressivos e traumatizantes de um hospital, visto que se constitui basicamente por pacientes considerados graves - o que exige demanda de cuidados especializados e de procedimentos técnicos de alta complexidade, com funcionamento ininterrupto por 24h durante os sete dias da semana, entre outras características (Santos \& Brasileiro, 2018; Fogaça, 2008). 
Neste contexto, exige-se do profissional de enfermagem tanto o investimento da energia física para a realização de um trabalho objetivo, como também energia psíquica, com importante envolvimento de subjetividades, alegrias, frustrações, queixas, sonhos e ideais. (Monteiro et al, 2013; Codo \& Vasques-Menezes, 1999).

Ajudar outras pessoas é reconhecidamente um objetivo nobre. No entanto, o exercício das profissões ditas de ajuda, pela natureza do seu trabalho e pelo impacto deste na vida dos trabalhadores, pode implicar em desgaste físico e emocional, insatisfação, falta de perspectiva, frustração e sentimentos de incompetência e fracasso os quais, em seu conjunto, identificam a síndrome de Burnout.

\section{Considerações Finais}

No presente estudo foi possível observar que apesar do trabalho ser considerado extenuante por alguns dos Enfermeiros pesquisados e existir indícios de esgotamento entre eles, não se identificou uma grande carga de exaustão profissional.

Da mesma forma, reconhece-se que há entre os participantes dessa pesquisa profissionais motivados e satisfeitos e, apesar do trabalho ser visto por alguns como desgastante, há, entre a maioria, um sentimento de satisfação em ser Enfermeiro e trabalhar com pessoas.

Sem encerrar qualquer uma das questões apresentadas, entende-se como importante a continuidade dessa pesquisa que poderá fornecer dados elucidativos para outros fatores, assim como subsidiar um planejamento em ações de Educação em Saúde, através do incremento de estratégias de prevenção à saúde do trabalhador da saúde. Pesquisas multicêntricas futuras são necessárias, para que se possa ter um panorama do estresse vivenciado pelos profissionais enfermeiros em diferentes cenários.

\section{Referências}

Barbosa, A. C. S., Luiz, F. S., Friedrich, D. B. C., Püschel, V. A. A., Farah, B. F., \& Carbogim, F. C. (2019). Profile of nursing graduates: competencies and professional insertion. Revista Latino-Americana de Enfermagem, 27, e3205. https://dx.doi.org/10.1590/1518-8345.3222.3205

Barboza, P. C., Pires A.S., Perez Junior E. F., Oliveira E. B., Santo, T. B. E., \& Gallasch, C. H. (2018). The meaning of work: perspectives of nursing professional who work in clinical units. Rev. Rene, (Vol. 19). http://dx.doi.org/10.15253/2175-6783.20181932819

Benevides-Pereira, A. M. T. (2014). Burnout: quando o trabalho ameaça o bem-estar do trabalhador. Casa do Psicólogo.

Braga, D. S., \& Paula, M. A. B. de. (2018). Síndrome de Burnout em profissionais de enfermagem. Rev. do Programa de Pós Graduação em Humanidades, Culturas e Artes UNIGRANRIO. 1(17).

Brasil. Conselho Nacional de Saúde. Resolução nº466 de 12 de dezembro de 2012. Diretrizes e Normas Regulamentadoras de Pesquisa em Seres Humanos. Diário Oficial da União, 12 dez. 2012.

Carlotto, M. S., \& Câmara, S. G. (2008). Análise da produção científica sobre a Síndrome de Burnout o Brasil. Psicol., 39(2): 152-158.

Codo, W., \& Vasques-Menezes, I. (1999). O que é Burnout. Em: CODO, W. (Org.). Educação: Carinho e trabalho.

Dalmolin, G. L., Lunardi, V. L., Lunardi, G.L., Barlem E. L. D., \& Silveira, R. S. (2014). Moral distresse and Burnout syndrome: are there relationship between these phenomena in nursing work? Rev Latino-Am Enfermagem, 22(1):35-42. http://dx.doi.org/10.1590/0104-1169.3102.23937.

Dutra, H. S., Gomes, P. A. L., Garcia, R. N., Oliveira, H. C., Freitas, S. C., \& Guirardello, E. B. (2019). Burnout entre profissionais de enfermagem em hospitais no Brasil. Revista Cuidarte, 10, 1, 1-13. http://dx.doi.org/10.15649/cuidarte.v10i1.585.

Estrela, C. (2018). Metodologia Científica: Ciência, Ensino, Pesquisa. Editora Artes Médicas.

Fogaça, M. C., Carvalho, W. B., Cítero, V. A., \& Martins, L. A. N. (2008). Fatores que tornam estressante o trabalho de médicos e enfermeiros em terapia intensiva pediátrica e neonatal: estudo de revisão bibliográfica. Rev. Bras. Ter. Intensiva, 20(3): 261- 6.

França, F. M. de, \& Ferrari, R. (2012). Síndrome de Burnout e os aspectos sócio-demográficos em profissionais de enfermagem. Acta Paulista de Enfermagem, 25(5), 743-748. https://dx.doi.org/10.1590/S0103-21002012000500015

Haddad, M. C. L., \& Jodas, D. A. (2009) Síndrome de Burnout em trabalhadores de enfermagem de um pronto socorro de hospital universitário. Acta Paul Enferm, 22(2): 192-7.

Lautert, L. (1997). O desgaste profissional: estudo empírico com enfermeiras que trabalham em hospitais. Revista Gaúcha de Enfermagem. $18,133-144$. 
Research, Society and Development, v. 10, n. 4, e38510414352, 2021

(CC BY 4.0) | ISSN 2525-3409 | DOI: http://dx.doi.org/10.33448/rsd-v10i4.14352

Machado, M. H. (Coord.) (2017). Perfil da enfermagem no brasil: relatório final. NERHUS - DAPS - ENSP/Fiocruz, 748 (Vol.01).

Maslach, C., \& Jackson, S. E. (1981). The measurementof experienced burnout. J Occup Behaviour, 2:99-113.

Monteiro, J. K., Oliveira, A. L. L. de, Ribeiro, C. S., Grisa, G. H., \& Agostini, N. de. (2013). Adoecimento psíquico de trabalhadores de unidades de terapia intensiva. Psicologia: Ciência e Profissão, 33(2), 366-379.

Perniciotti, P., Serrano Júnior, C. V., Guarita, R. V., Morales, R. J., \& Romano, B. W. (2020). Síndrome de Burnout nos profissionais de saúde: atualização sobre definições, fatores de risco e estratégias de prevenção. Revista da SBPH, 23(1), 35-52. http://pepsic.bvsalud.org/scielo.php?script=sci_arttext\&pid=S1516-08582020000100005\&lng=pt\&tlng=pt

Rodrigues, C. C. F. M., Santos, V. E. P., \& Sousa, P. (2017). Segurança do paciente e enfermagem: interface com estresse e Síndrome de Burnout. Revista Brasileira de Enfermagem,70(5),1083-88. http://www.scielo.br/pdf/reben/v70n5/pt_0034-7167-reben-70-5-1083.pdf.

Rosa, C., \& Carlotto, M. S. (2005). Síndrome de burnout e satisfação no trabalho em profissionais de uma instituição hospitalar. Rev. SBPH, 8(2): 1-15.

Sá, A. M. S., Martins-Silva, P. O., \& Funchal, B. (2014). Burnout: o impacto da satisfação no trabalho em profissionais de enfermagem. Psicologia \& Sociedade, 26(3), 664-674. https://doi.org/10.1590/S0102-71822014000300015.

Santos, G. W. dos, Cargnin, M. C. dos S., Tasqueto, J. V. G., Oliveira, B. de, \& Hausmann, O. I. (2020). Factors associated with Burnout Syndrome in nursing professionals. Research, Society and Development, 9(3), e117932574. https://doi.org/10.33448/rsd-v9i3.257

Santos, I. M., \& Brasileiro, M. E. (2018). Síndrome de Burnout em Profissionais de Enfermagem: dos Fatores de Riscos à Estratégias de Prevenção. Revista Científica Multidisciplinar Núcleo do Conhecimento, (6a ed.), 3(2), 84-112.

Silva, F. G., Andrade, A. P., Ponte, K. M. A., Ferreira, V. E. S., Sousa, B. S., \& Gonçalves, K. G. (2019). Predisposição para síndrome de burnout na equipe de enfermagem do serviço de atendimento móvel de urgência. Enfermagem em Foco, 10(1),40-45. http://revista.cofen.gov.br/index.php/enfermagem/article/view/1600

Silva, K. S. G. et al. (2020). A síndrome de Burnout em profissionais de enfermagem. ReBIS, 2(1), 38-42. http://revistarebis.rebis.com.br/index.php/rebis/article/view/67/61.

Tamayo, M., \& Tróccoli, B. T.(2002). Burnout no trabalho. In: Mendes, A. M., Borges, L. O., Ferreira, M. C., (Org). Trabalho em transição, saúde em risco. Brasília Ed. UNB, 45-63. 